\title{
Primary Liver Sarcomatoid Carcinoma: A Case Series and Literature Review
}

\author{
Wei Ji (1D' \\ Yunlong Xing ${ }^{2}$ \\ Jinshu $\mathrm{Ma}^{3}$ \\ Zhuo Zhao ${ }^{4}$ \\ Hongqin $\mathrm{Xu}^{5}$ \\ Shuang Zheng ${ }^{6}$ \\ Wei $\mathrm{Li}^{1}$ \\ $\mathrm{Xu} \mathrm{Li}$ \\ 'Cancer Center, The First Hospital of Jilin \\ University, Changchun, People's Republic \\ of China; ${ }^{2}$ Plastic Surgery, China-Japan \\ Union Hospital, Jilin University, \\ Changchun, People's Republic of China; \\ ${ }^{3}$ Department of Pathology, China-Japan \\ Union Hospital, Jilin University, \\ Changchun, People's Republic of China; \\ ${ }^{4}$ Cardiology Department, The Second \\ Hospital of Jilin University, Changchun, \\ People's Republic of China; ${ }^{5}$ Department \\ of Hepatology, The First Hospital of Jilin \\ University, Changchun, People's Republic \\ of China; ${ }^{6}$ Department of Radiology, The \\ First Hospital of Jilin University, \\ Changchun, People's Republic of China
}

Correspondence: $\mathrm{Xu} \mathrm{Li}$

Department of Hepatology, The First

Hospital of jilin University, No. 7I, Xinmin

Street, Changchun, I3002I, People's

Republic of China

Tel +86I58 0430 I765

Email jdyylx@jlu.edu.cn
Background: Primary liver sarcomatoid carcinoma (PLSC) is rare. To improve the understanding of PLSC, cases were described and reviewing the literature.

Methods: A retrospective analysis was performed on 14 cases of PLSC diagnosed by pathology in Northeastern China from 2010 to 2020. An individual participant data analysis based on reported cases was conducted to determine epidemiological characteristics, clinical characteristics, and prognoses of PLSC.

Results: A total of 136 cases involved our 14 cases and 122 cases from previous reports. The percentages of sarcomatoid hepatocellular carcinoma, sarcomatoid cholangiolocellular carcinoma, and mixed and unclassified types were $36.8 \%, 41.9 \%, 5.9 \%$, and $15.4 \%$, respectively. A total of 95.6\% PLSC was found in Asia. There was a lower percentage of hepatitisinfected patients in Japan, when compared with the Republic of Korea (38.5\% vs $70.0 \%$, $\mathrm{P}<0.05$ ). Five cases were initially misdiagnosed as a hepatic abscess by imaging. A total of $36.7 \%$ cases had metastases when being diagnosed, and $68.9 \%$ cases relapsed during the follow-up. The median disease-free survival and overall survival (OS) were 3 months and 5 months, respectively. Only radical surgery (hazard ratio $=0.308,95 \%$ confidence interval $0.186-0.512, \mathrm{P}<0.001$ ) indicated a better OS.

Conclusion: PLSC was more prevalent in Asia and there were possibilities of misdiagnoses. Surgery is still an effective treatment and can significantly prolong the OS. Only limited strategies for recurrent or advanced PLSC, immunotherapy may be possible treatment.

Keywords: liver, sarcomatoid carcinoma, pathology, immunotherapy

Primary liver sarcomatoid carcinomas (PLSCs) are tumors containing both malignant epithelial and spindle cell sarcomatoid components, including those of hepatocellular origin, cholangiolocellular origin, and mixed and unclassified origin. ${ }^{1}$ PLSC is rare but aggressive. Jernigan et $\mathrm{al}^{2}$ reported that only $2.6 \%(20 / 784)$ of primary liver cancers (PLCs) were combined with spindle-shaped cells. A total of 1.8\% 9.4\% of hepatocellular carcinomas (HCCs) have sarcomatoid changes, and sarcomatoid cholangiolocellular carcinomas (SCCs) account for less than 1\% of intrahepatic cholangiolocellular carcinomas (CCs). ${ }^{3}$ Only 122 cases of PLSC have been reported since the definition was proposed by the World Health Organization (WHO) in 2000. The pathogenesis of PLSC is not definitively known. Anti-tumor treatment histories, such as transarterial chemoembolization (TACE), radiofrequency ablation, and percutaneous ethanol injection have been considered as inducements or accelerators of sarcomatous change, although this possibly has not been supported by clinical cases. ${ }^{4,5}$ The current thought is that sarcomatoid differentiation occurs spontaneously during tumor genesis. No specific features have been identified in the symptoms, or in the serological and imaging 
findings. A pathological test is the only method to diagnose PLSC, so the diagnosis should be conducted with caution, especially in needle biopsy specimens. However, immunohistochemistry (IHC) may provide additional information to improve the diagnosis. PLSC is prone to relapse and has a worse prognosis, and evidence-based therapy of PLSC is still insufficient. Characterization of immune infiltration in PLSC were revealed and few cases have tried immunotherapy recently, which shed new light on the treatment dilemma. In the present study, we therefore described 14 cases of PLSC diagnosed during a 10-year period, and reviewed PLSCs to analyze the features, which could be inspirational in peer communications.

\section{Materials and Methods Our Cases}

This study was conducted in accordance with the Declaration of Helsinki and was approved by the Ethical Committee of the First Hospital of Jilin University. Written informed consents were obtained from the patients or their lineal relations. Clinical information of 14 PLSC cases diagnosed according to their pathologies in Northeastern China between 2010 and 2020 were retrospectively collected, including basic characteristics, laboratory tests, imaging results, pathology data, and IHC staining results. Follow-up was conducted by phone to obtain treatment methods and outcomes of the patients. Disease-free survival (DFS) was recorded as the time period from surgery to tumor recurrence or death for any reason (for patients receiving radical surgeries). Overall survival (OS) was recorded as the time period from diagnosis to death for any reason.

\section{Diagnostic Criteria of PLSC}

According to the definition from the WHO: ${ }^{1}$ (1) the tumors contain both carcinomatous components and sarcomatous components, (2) tumors show cell morphologies of sarcomatous components varying from spindled to epithelioid and pleomorphic, and (3) positive epithelial and mesenchymal markers, using IHC staining, are present.

\section{Eligibility Criteria and the Search Strategy}

Published case reports of proven/probable PLSC cases were reviewed. The search items were ("liver"'[MeSH Terms] OR "liver"[All Fields] OR "livers"[All Fields] OR "liver s"[All Fields] OR ("hepatic"[All Fields] OR "hepatophyta"[MeSH Terms] OR "hepatophyta"[All Fields] OR "hepatics"[All Fields]) OR "hepatocellular”[All Fields]) AND
("sarcomatoid"[All Fields] OR ("carcinosarcoma"[MeSH Terms] OR "carcinosarcoma"[All Fields] OR “carcinosarcomas"[All Fields])) AND 2000/01/01:2021/06/ 31[Date - Publication] in PubMed. The reference lists of included studies and related publications were also screened to identify relevant studies. The results were then searched by hand and screened. Studies passing the screening list were then added.

\section{Statistical Analysis}

Statistical analysis was performed using SPSS statistical software for Windows, version 26.0 (SPSS, Chicago, IL, USA) (IBM SPSS Statistics, RRID:SCR_019096)) for pooled cases, including the cases previously reported and 14 cases reported in the present study. The cases were divided into four groups according to the pathological diagnoses as sarcomatoid hepatocellular carcinoma (SHC), SCC, mixed, or unclassified types. Categorized data were analyzed using the chi-square test. Kaplan-Meier analysis and the Log rank test were used for survival analyses. The Cox proportional hazards regression model was used to identify independent prognostic factors of survival. A value of $\mathrm{P}<0.05$ was assumed to be statistically significant.

\section{Results}

\section{Clinical Materials of the I4 PLSC Cases Basic Characteristics and Clinical Findings}

Among the 14 cases, 12 cases $(85.7 \%)$ were male. The median age was 61.5 years. Six cases $(42.9 \%)$ were associated with hepatitis B and/or hepatitis C. Only one case had a history of anti-tumor treatment. No aggravated increase of serum aspartate aminotransferase (AST) and alanine aminotransferase (ALT) levels ( $>2$-fold of the upper limit of reference) were observed. Seven of 14 patients (50\%) had elevated gamma-glutamyl transferase ( $>2$-fold of the upper limit of reference), but only one patient had mildly elevated direct-bilirubin of $16.0 \mu \mathrm{mol} / \mathrm{L}$ (reference: $<6.8 \mu \mathrm{mol} / \mathrm{L}$ ). The carcinoembryonic antigen of all 14 patients were in the normal range. Only one patient had a $>2$-fold increased level of alpha-fetoprotein with $113.8 \mu \mathrm{g} / \mathrm{L}$ (reference: $<7 \mu \mathrm{g} / \mathrm{L}$ ), who also had hepatitis B+C. Four patients had increased carbohydrate antigen 19-9 (CA19-9), but only one patient surpassed the 2-fold reference of CA19-9 with $51.2 \mu \mathrm{g} / \mathrm{L}$ (reference: $<19 \mu \mathrm{g} / \mathrm{L}$ ) (Table 1). The imaging results were available in 11 cases. Among them, five cases (Cases 8, 11, 12,13 , and 14) were considered as "hepatic abscess probable" by imaging, and ultrasound (US)-guided percutaneous 


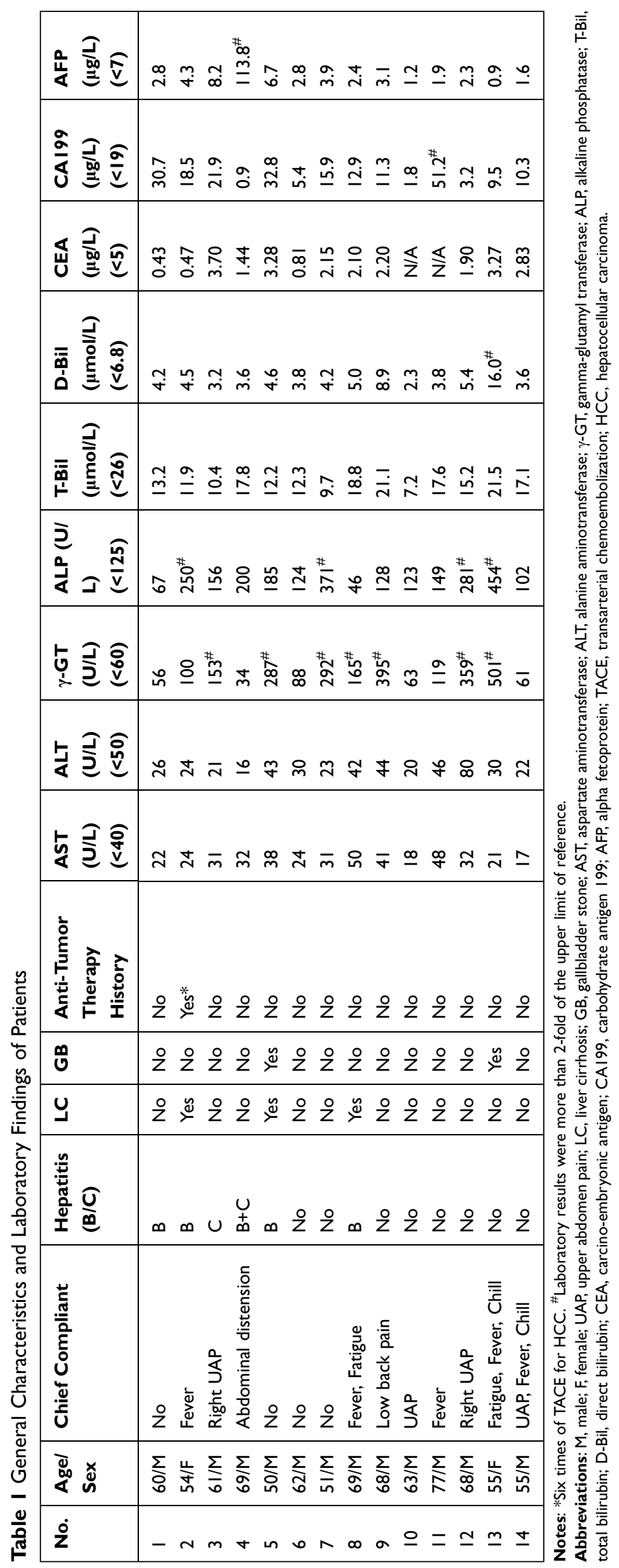


drainages for hepatic abscesses were performed for Cases 11 and 12. Representative images of computed tomography (CT) and magnetic resonance imaging (MRI) are shown in Supplementary 1.

\section{Pathological Findings}

Four biopsy tissues and all seven pieces of postoperative tissues had necrotic regions, including one lesion with mass hemorrhage. The maximum diameter of tumors was greater than $5 \mathrm{~cm}$ in all cases except Case $6(2.7 \mathrm{~cm})$ and Case $9(4.6 \mathrm{~cm})$, and three cases with multiple lesions. Pathological examinations of all tissues showed double positives of at least one marker from epithelial components (CK, CK-Pan, CK7, and EMA, etc.) and one marker from sarcomatous components (vimentin, CD34, and SMA, etc.). Ki-67 was found in 10 cases with positive percentages from $30 \%$ to $90 \%$. The pathology and IHC findings are summarized in Supplementary 2.

\section{Treatments and Outcomes}

The follow-up percentage of $57.1 \%$ (8/14). Five cases were not treated. Radical surgery was performed on seven cases, with one case (Case 5) adopting TACE shortly after surgery. Case 1 obtained a 13.5-month DFS and two times of radiofrequencies were adopted to deal with the single relapse lesion. Then Sorafenib was adopted for a multi-lesion relapse, which last for 3.5 months until mortality. The OS was 23.2-month and the treatment process is shown in Figure 1. Case 4 obtained a 1.7-month DFS and adopted two cycles of chemotherapy (epirubicin + cisplatin) after relapse but discontinued due to myelosuppression. Case 9 was at stage IVB when diagnosis, but docetaxel combined with cisplatin were adopted only one cycle due to personal reasons, whose OS is 2.8 months. Treatments and clinical outcomes are listed in Supplementary 3. The median DFS was not determined, and the median OS of all cases was 16.8 months.

\section{Literature Review}

A total of 480 articles were found, and 49 articles involving 122 cases were ultimately included using the steps shown in Figure 2. A list of published case reports/ series included in the review was in Supplementary 4.

\section{Basic Characteristics of Tumors of Different Origin}

Among 136 cases including 122 from literature review and 14 in this study, 50 cases $(36.8 \%)$ were SHC, 57 cases $(41.9 \%)$ were SCC, eight cases (5.9\%) were mixed, and 21 cases (15.4\%) were of unclassified origin. The ratio of the old ( $>$ 60 years, 64 cases) and the young ( $\leq 60$ years, 71 cases) was $0.90 .49 .3 \%$ cases $(67 / 136)$ had hepatitis and anti-tumor treatment history was only reported in 12 cases $(8.8 \%)$. Sex, age, and liver cirrhosis were not associated with different pathological types $(\mathrm{P}>0.05)$. Geographic variation was also observed, with $95.6 \%$ cases reported from Asian countries, and only $4.4 \%$ from Europe. The percentages of patients with or without hepatitis were similar $(55.4 \%$ vs $44.6 \%$, respectively). Patients with hepatitis were more vulnerable with SHC (30.6\%) $(\mathrm{P}<0.05)$, which was less prevalent in patients without hepatitis $(6.6 \%)(\mathrm{P}<0.05)$ (Table 2). In total, $70 \%$ of the cases were combined with hepatitis in the Republic of Korea,
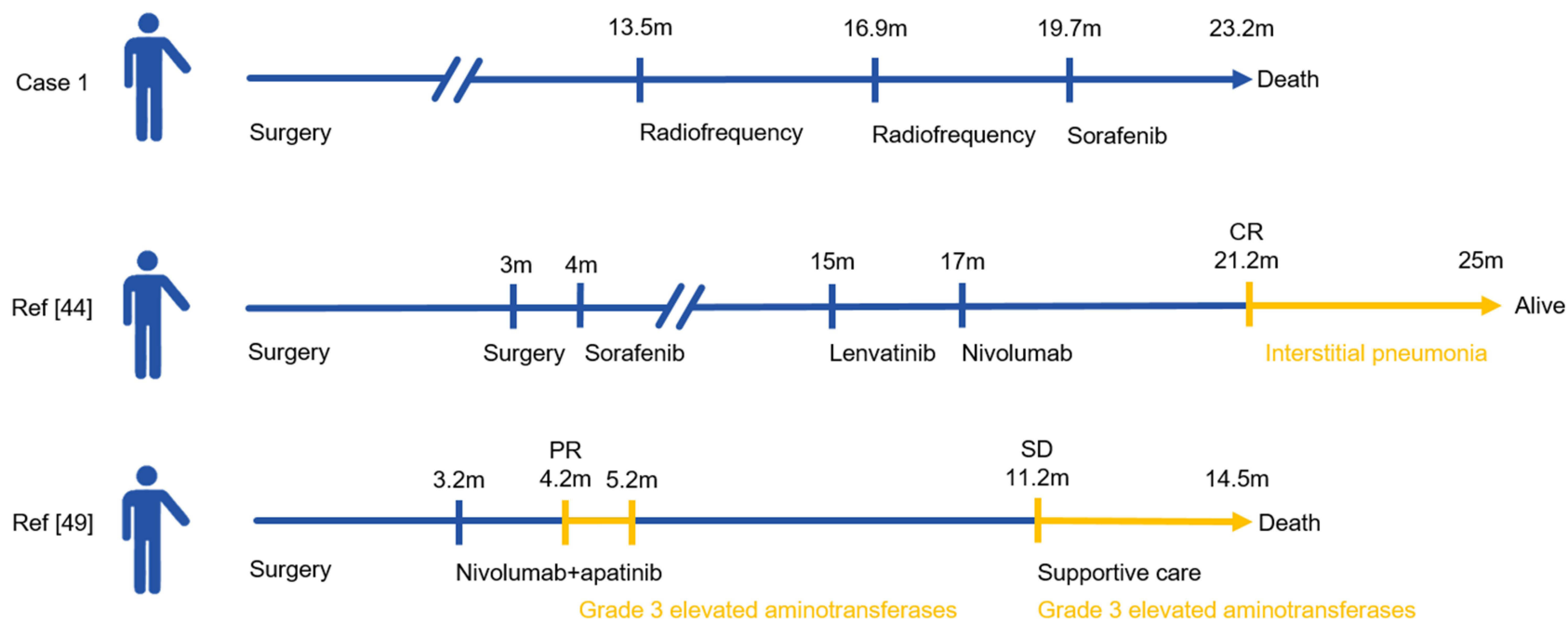

Figure I The treatment process.

Note: The yellow line represents no anti-tumor therapy and the reasons for the decisions.

Abbreviations: CR, completed regression; PR, partial regression; SD, stable disease. 


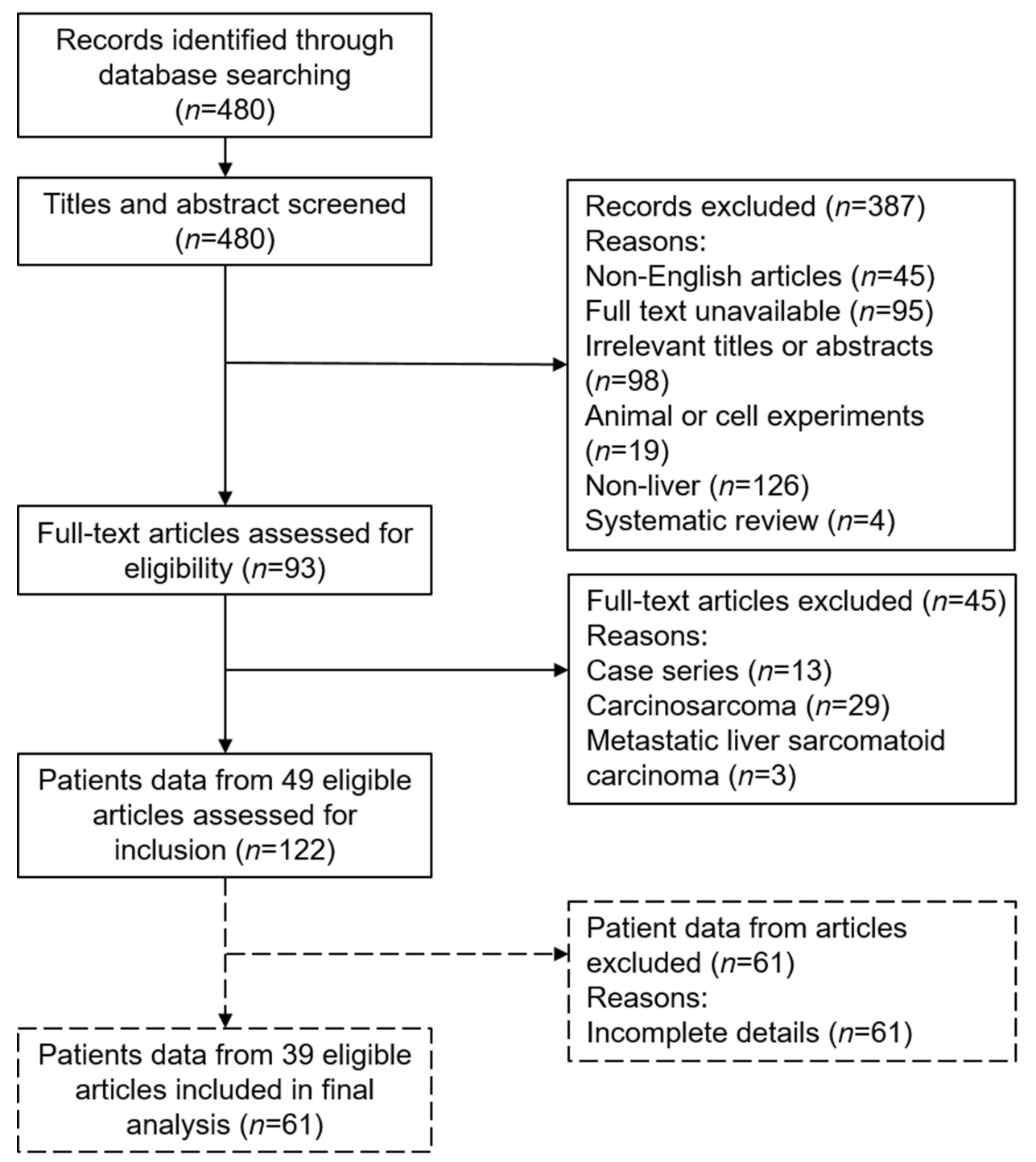

Figure 2 Flowchart of literature screening.

but only $38.5 \%$ in Japan $(\mathrm{P}<0.05)$. More details are shown in Supplementary 5.

\section{Biological Characteristics of PLSCs}

The median tumor size was $7.80 \mathrm{~cm}$ in a total of 136 cases. Then, 61 cases from 10 studies were excluded for incomplete details as described in Figure 1. Among 75 cases with complete details, only 11 cases $(14.7 \%)$ reported satellite nodules, 29 cases $(38.6 \%)$ had metastases when diagnosed, and 51 cases $(68.0 \%)$ relapsed during the follow-up (Supplementary 6). Also, three cases reported misdiagnosis as abscess ( 1 case in ARTICLE 38, and cases in ARTICLE 43 and ARTICLE 44 listed in Supplementary 4).

\section{Survival Analysis}

Survival analyses were conducted in all 136 cases, with the median DFS and OS being 3.0 months and 5.0 months, respectively. Using univariate analyses, age and pathological types were not associated with either the DFS or OS $(\mathrm{P}>$ 0.05 ), and TNM stages and anti-tumor treatments were associated with the OS, but not with the DFS. Patients who received surgery had a median OS of 12.0 months [95\% confidence interval (CI): 8.62-15.30) (Table 3). All parameters were then analyzed using Cox regression. Only the anti-tumor treatment was found to be a prognostic factor (Figure 3). Surgery significantly prolonged the OS with hazard ratio $(\mathrm{HR})=0.308(95 \% \mathrm{CI} 0.186-0.512, \mathrm{P}<0.001)$ (Figure 4$)$. 
Table 2 Summary of Cases from the Literature Review and This Study $(n=129)$

\begin{tabular}{|c|c|c|c|c|c|c|c|}
\hline Features & Total & $\operatorname{SHC}^{a}(n=46)$ & $\operatorname{SCC}^{b}(n=54)$ & $S H C+C C^{c}(n=8)$ & $\operatorname{uPLSC}^{d}(n=21)$ & $\chi^{2}$ & $P$ \\
\hline Sex & & & & & & 1.598 & 0.654 \\
\hline Male & $99(72.8)$ & $37(27.2)$ & $43(31.6)$ & $6(4.4)$ & $13(9.6)$ & & \\
\hline Female & $37(27.2)$ & $13(9.6)$ & $14(10.3)$ & $2(1.5)$ & $8(5.9)$ & & \\
\hline Age & & & & & & 2.925 & 0.392 \\
\hline$>60$ & 64(47.4) & $19(14.1)$ & $30(22.2)$ & $4(3.0)$ & II (8.I) & & \\
\hline$\leq 60$ & 7I(52.6) & $3 I(23.0)$ & $26(19.3)$ & $4(3.0)$ & $10(7.4)$ & & \\
\hline Region & & & & & & 2.440 & 0.455 \\
\hline Asia & $130(95.6)$ & $48(35.3)$ & $54(39.7)$ & $7(5.1)$ & $21(15.4)$ & & \\
\hline Europe & $6(4.4)$ & $2(1.5)$ & $3(2.2)$ & $I(0.7)$ & $0(0)$ & & \\
\hline Hepatitis & & & & & & 24.654 & $<0.001$ \\
\hline Yes & $67(55.4)$ & $37(30.6)^{b, d}$ & $17(14.0)$ & $5(4.1)$ & $8(6.6)$ & & \\
\hline No & $54(44.6)$ & $8(6.6)$ & $34(28.1)^{\mathrm{a}}$ & $3(2.5)$ & $9(7.4)^{\mathrm{a}}$ & & \\
\hline Liver cirrhosis & & & & & & 2.545 & 0.486 \\
\hline Yes & $30(33.3)$ & $10(11.1)$ & $\mathrm{II}(\mathrm{I} 2.2)$ & $4(4.4)$ & $5(5.6)$ & & \\
\hline No & $60(66.7)$ & $17(18.9)$ & $28(3 I .1)$ & $3(3.3)$ & $12(13.3)$ & & \\
\hline
\end{tabular}

Notes: Column proportion test (z-test) assigned key letter ${ }^{a}$ b, c, d to SHC, SCC, SHC+CC and uPLSC, separately. And put the key letters with a smaller proportion of categories aside by the category with a larger proportion.

Abbreviations: SHC, sarcomatoid hepatocellular carcinoma; SCC, sarcomatoid cholangiocellular carcinoma; uPLSC, unclassified primary liver sarcomatoid carcinoma.

Table 3 Univariate Analysis for Survival of Cases from the Literature Review and This Study $(n=129)$

\begin{tabular}{|c|c|c|c|c|c|c|}
\hline Features & mDFS & $\chi^{2}$ & $P$ & mos & $\chi^{2}$ & $P$ \\
\hline All cases & $3.0(2.02-3.98)$ & & & $5.0(2.86-7.14)$ & & \\
\hline $\begin{array}{l}\text { Age } \\
\qquad 60 \\
\leq 60\end{array}$ & $\begin{array}{l}3.0(1.51-4.50) \\
3.0(1.27-4.73)\end{array}$ & 0.007 & 0.935 & $\begin{array}{c}8.0(3.35-12.65) \\
4.0(2.79-5.20)\end{array}$ & 1.744 & 0.187 \\
\hline $\begin{array}{l}\text { Diagnosis } \\
\text { SHC } \\
\text { SCC } \\
\text { SHC+CC } \\
\text { uPLSC }\end{array}$ & $\begin{array}{c}3.0(1.64-4.36) \\
3.0(0.76-5.24) \\
8.0(1.76-14.24) \\
3.0(0.43-5.57)\end{array}$ & 0.907 & 0.824 & $\begin{array}{c}11.0(7.15-14.85) \\
3.0(2.55-3.45) \\
24.0 \\
5.0(2.92-7.09)\end{array}$ & 6.771 & 0.052 \\
\hline $\begin{array}{l}\text { TNM Stage } \\
\qquad \begin{array}{l}I+I \\
I I I+I V\end{array}\end{array}$ & $\begin{array}{l}5.0(2.55-7.45) \\
2.0(I .29-2.71)\end{array}$ & 3.138 & 0.076 & $\begin{array}{c}\mid \mathrm{I} .0(5.79-16.21) \\
4.6(3.82-5.38)\end{array}$ & 6.364 & 0.012 \\
\hline $\begin{array}{l}\text { Anti-tumor treatment } \\
\text { Surgery } \\
\text { Others } \\
\text { No }\end{array}$ & $\begin{array}{c}4.0(2.90-5.10) \\
3.0(1.83-4.17) \\
\text { N/A }\end{array}$ & 0.385 & 0.535 & $\begin{array}{c}12.0(8.62-15.30) \\
4.3(3.86-4.74) \\
2.0(1.92-2.08)\end{array}$ & 27.614 & $<0.001$ \\
\hline
\end{tabular}

Abbreviations: m, median; DFS, disease-free survival; OS, overall survival; SHC, sarcomatoid hepatocellular carcinoma; SCC, sarcomatoid cholangiocellular carcinoma; uPLSC, unclassified primary liver sarcomatoid carcinoma; N/A, not applicable.

\section{Discussion}

PLSC has a relatively short history. In 2000, SHC was first mentioned as a type of digestive system tumor by the WHO, who stated that, "When such sarcomatoid features are prominent, the tumor is called sarcomatoid HCC." However,
Torbenson ${ }^{6}$ reported that $1 \%$ to approximately $80 \%$ of the spindle-shaped cells without an epithelial or mesenchymal origin were found in PLSCs. The definition of PLSC has now been acknowledged by investigators as tumors containing both carcinomatous (either hepatocellular or 


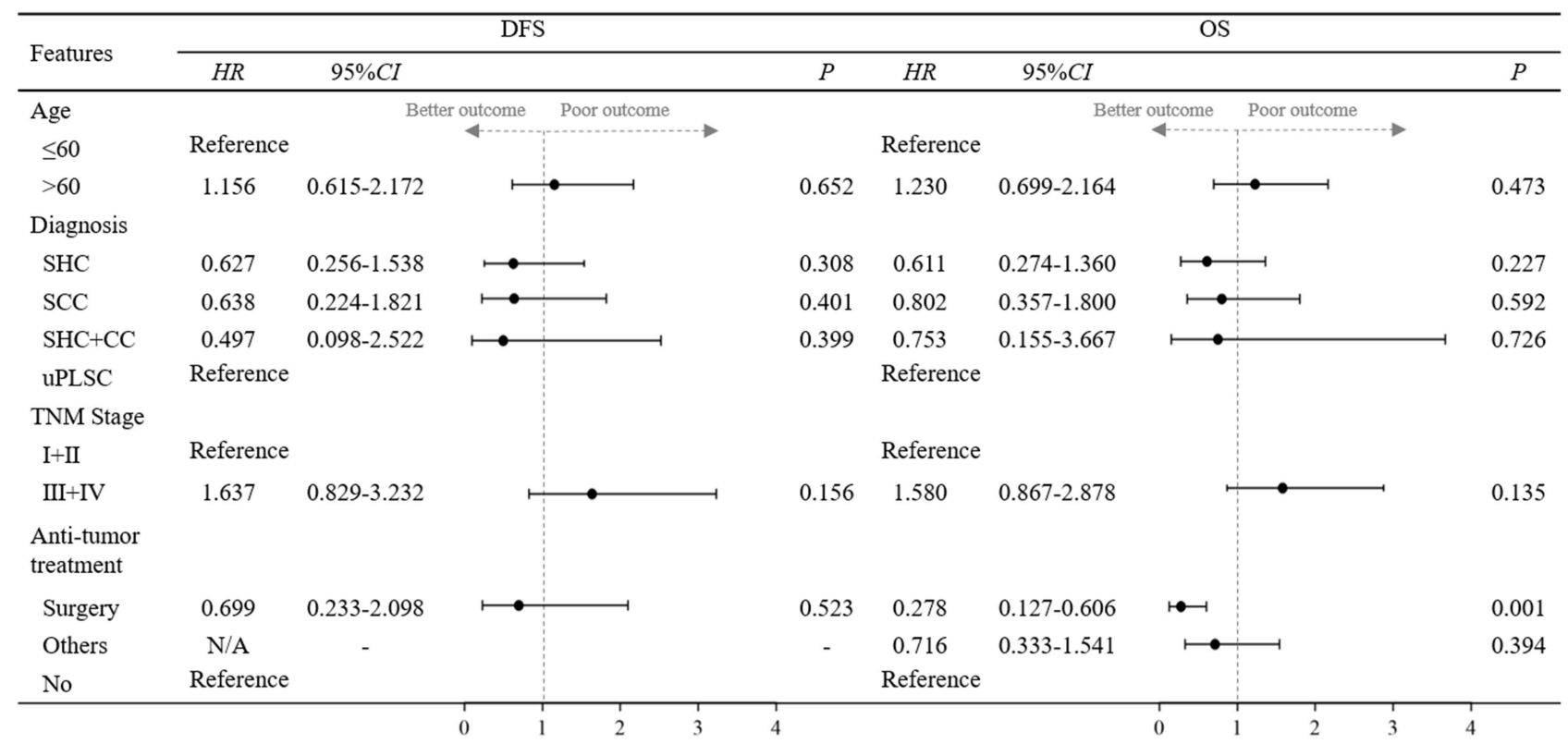

Figure 3 Multivariate analysis and a forest plot for survival of cases from the literature review and from this study paper ( $\mathrm{n}=136$ ).

Abbreviations: DFS, disease-free survival; OS, overall survival; SHC, sarcomatoid hepatocellular carcinoma; SCC, sarcomatoid cholangiocellular carcinoma; uPLSC, unclassified primary liver sarcomatoid carcinoma; N/A, not applicable.

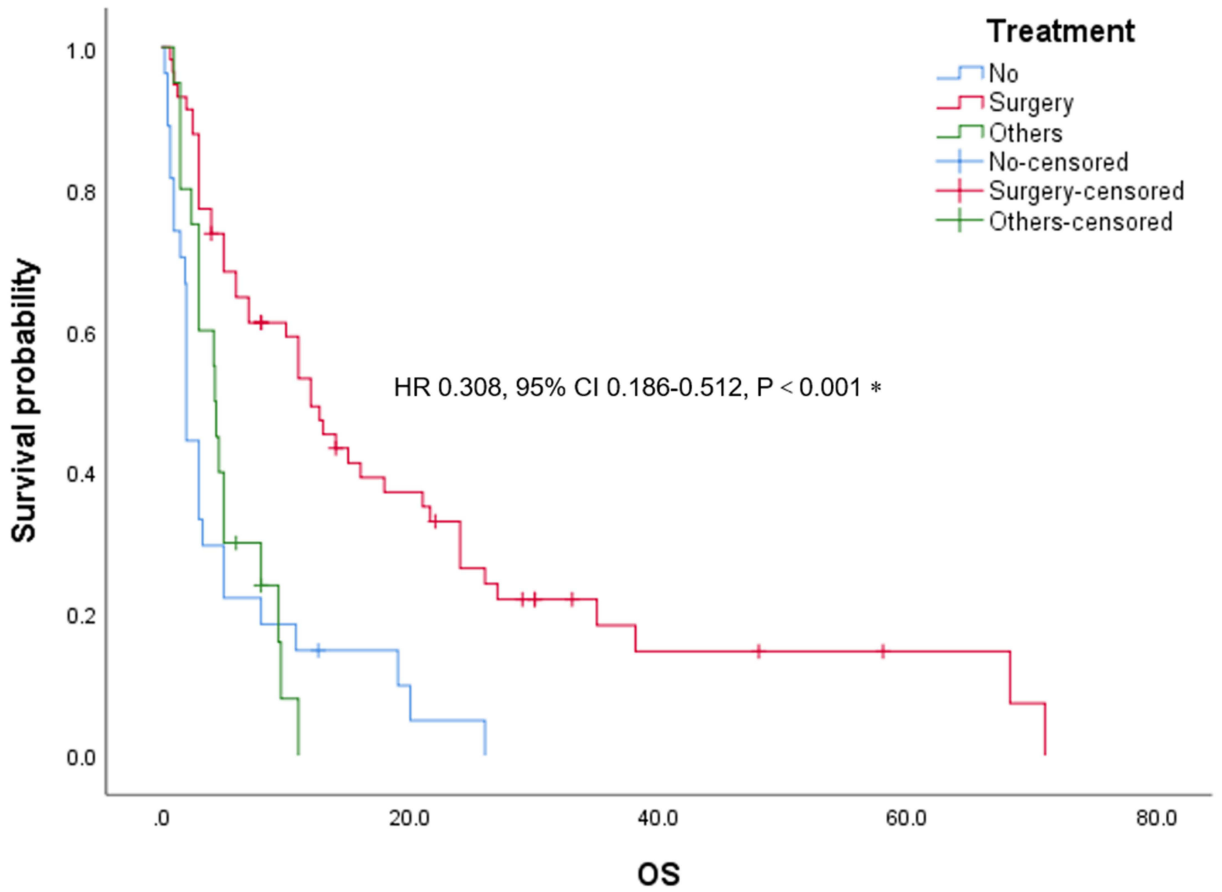

Figure 4 Kaplan-Meier survival analysis of overall survival according to treatment strategies in patients.

Note: *Using the no-treatment group as a reference.

Abbreviation: OS, overall survival.

cholangiolocellular) and sarcomatous components. Cells of sarcomatous components are atypical due to dedifferentiation. If the sarcomatous components contain variable malignant mesenchymes such as chondrosarcomas, rhabdomyosarcomas, or osteosarcomas, the tumor is classified as a carcinosarcoma. $^{7}$ In addition, spindle-shaped or 
polymorphic cells of sarcomatous components must be double positive for markers of the epithelium and mesenchyme. Moreover, IHC of epithelial markers is useful for clarifying the origin, such as heppar-1, CK7, and CK19, to classify PLSCs into SHC, SCC, and mixed and unclassified types.

PLSC is reported to be more common in older males, who account for $73-82.5 \%$ of cases. ${ }^{8-10}$ In the 136 pooled cases, $72.8 \%$ of PLSC cases were male, but there were minor differences between old and young aged cases with a ratio of 0.90 . Hepatitis is considered as a possible risk factor, ${ }^{10}$ but not drinking, the anti-tumor treatment history, or gallbladder stones. ${ }^{1-14}$ A significantly higher incidence of SHC with hepatitis has been reported, which is similar to the epidemiological characteristics of $\mathrm{HCC}$, indicating the tumorigenesis of SHC. ${ }^{15,16}$ Another notable observation has been that PLSC is prevalent in Asian countries, especially in China, the Republic of Korea, and Japan, ${ }^{17}$ but there are less cases infected with hepatitis in Japan. A 29-year-old PLSC patient has been reported with a 10-year history of schistosomiasis, who was negative for hepatitis B and C. ${ }^{9}$ Besides PLSC, schistosomiasis infection was also reported in bladder sarcomatoid carcinoma. ${ }^{18,19}$ Thus, schistosomiasis cannot be ruled out as a risk factor. However, only a limited number of cases have mentioned schistosomiasis, so this possible association needs further investigation.

The pathogenesis and histogenesis of sarcomatoid carcinoma have not been determined. The "collision hypothesis" has suggested the emergence of tumors from the epithelium and mesenchyme, ${ }^{20}$ which has been denied by the unclear transitional zone between carcinomatous and sarcomatous components. However, there are still cases, which have reported a clearly defined HCC and sarcomatous components without an area of overlap. ${ }^{20}$ The epithelial-mesenchymal transition (EMT) is an epigenetic process. During the EMT, cells change into a spindle-shaped morphology and express mesenchymal cell markers, notably vimentin and $\mathrm{N}$-cadherin. The EMT promotes the "invasion and metastasis cascade" 21 and acquisition of stem cell traits, ${ }^{22}$ which partially account for relapses and a poor prognosis. Another possibility is simultaneous malignant transformation from pluripotent stem cells to both epithelial and mesenchymal cells. $^{23,24}$ Conventional neoplastic cells are capable of transforming into multipotent immature cells and then, sarcomatoid redifferentiation occurs, ${ }^{25,26}$ which is especially suitable for mixed histopathological findings of SHC $+\mathrm{CC}$. Cases originating from normal hepatocytes without cirrhosis also support the pluripotent precursor cell or stem cell hypothesis. $^{27} \mathrm{Gu}$ et $\mathrm{al}^{28}$ analyzed the fractional allelic loss (FAL) index of three SHCs, to show a polyclonal origin for all three cases (the FALs were 50.0\%, 55.6\%, and 33.9\%). Stochastic phenotype switching during mitosis also contributes to intratumoral heterogeneity in PLC. ${ }^{29}$

Pathological analysis is presently the method of choice for diagnosis. However, clinical misdiagnosis of PLSC is possible due to atypical symptoms and serological and radiological results. ${ }^{9,10,30-32}$ Leukemoid reaction has also been reported as the earliest manifestation, which makes the diagnosis more confusing. ${ }^{33,34}$ Satellite nodules with similar radiological features as the main lesion may also be a clue to the presence of PLSC. ${ }^{35}$ However, only $14.7 \%$ of cases have reported satellite nodules, so it is not useful in clinical practice. In PLSC patients, $44.0 \%$ cases have necrosis and even hemorrhages. No enhancements of inside areas due to necrosis are common using CT and MRI, so it is easily confused with a hepatic abscess. In diffusion weighted imaging (DWI) mapping, multiple cystic changes accompanied by fibrous septum exhibit nonhomogeneous and hyperintense changes in the center, which is also similar to an atypical liver abscess. ${ }^{15}$ Drainage in misdiagnosed cases has occurred in clinical practice as previous reports by Wang et $\mathrm{al}^{36}$ and Shi et $\mathrm{al}^{37}$ including two cases in this study (Cases 11 and 12). After investigating 6901 cases of malignant liver tumors, Seo et $\mathrm{al}^{38}$ reported that PLSC patients had a greater prevalence of rim enhancements than PLC patients. Contrast-enhanced ultrasound detects a thicker ring-enhancing part of the abscess than that of PLSC patients, ${ }^{39}$ peripheral nodular enhancement of PLSC, and hypervascularization of HCC in the arterial phase. ${ }^{31,40}$ However, these differences are minor and difficult to practice, so difficulties in diagnoses should always be considered.

PLSC is invasive and frequently relapses. According to our results, the recurrence percentage was $68.0 \%$, which is consistent with the high index of Ki-67 (30-90\%). The prognosis is also poor. Okabayashi et $\mathrm{al}^{8}$ reported that survival was $46.0 \%$ during the first year and $9.9 \%$ until the fifth year after surgery. Data from 136 cases showed that the median DFS was 3 months and the median OS was only 5 months. There is no guideline for the treatment of PLSC. Radical surgery is currently recognized as the most effective treatment option and significantly prolongs the OS $(\mathrm{HR}=0.308,95 \%$ CI $0.186-0.512, \mathrm{P}<0.001)$. Liver transplantation for SHC patients has also achieved a similar prognosis as liver resection. ${ }^{41}$ Adjuvant chemotherapy, involving 5-fluorouridine combined with cisplatin, has also been used 
in two cases. One case soon relapsed with a DFS of 3 months, and the other case had a DFS of more than 6 months. ${ }^{14,42}$ Thus, it is difficult to make a definitive conclusion about the efficacy of adjuvant chemotherapy.

For patients suffering from advanced PLSC at initial diagnosis and with recurrence after surgery, the prognosis is worse, with a median OS of less than 6 months. ${ }^{43}$ Cytotoxicity drugs and sorafenib ${ }^{44,45}$ have been used for treatments, including gemcitabine combined with paclitaxel, ${ }^{35}$ gemcitabine combined with cisplatin, ${ }^{40}$ and pirarubicin combined with cisplatin, ${ }^{46}$ but only in case reports. Epirubicin combined with cisplatin (Case 4) and docetaxel combined with cisplatin (Case 9) have been used in two of our cases, but unplanned terminations made it impossible to provide information about the treatment-related prognoses. Apatinib, a vascular endothelial growth factor receptor 2 inhibitor, is effective for both advanced PLC (NCT01192971) and carcinosarcoma (NCT03064243). Although it was reported that vascular invasion was a prognosis factor of OS with $\mathrm{HR}=4.931$ (95\% CI 1.190 20.430, $\mathrm{P}=0.028) .{ }^{47}$ There is insufficient data to determine whether treatment with apatinib results in clinical benefit for PLSC patients. Case 10 in the present study was treated with apatinib after a recurrence, but the OS was only 4.4 months.

With breakthroughs in immunotherapy for solid tumors, the use of immunotherapy combined with chemotherapy and (or) targeted therapy has also been reported. ${ }^{48}$ Zhao et $\mathrm{al}^{49}$ reported that a patient weakly positive for programmed cell death ligand-1 (PD-L1) relapsed after surgery, while treatment with nivolumab (3 $\mathrm{mg} / \mathrm{kg}$ biweekly) combined with apatinib resulted in a partial response after four cycles, but resulted in grade 3 elevated aminotransferase levels, with an OS of 12 months, which was similar with that of patients receiving radical surgery (Figure 1). Zhu et $\mathrm{al}^{44}$ reported a patient who relapsed after surgery with high expression of PD-L1 [the tumor proportion score (TPS) was $60 \%$ ] treated with nivolumab (3 $\mathrm{mg} / \mathrm{kg}$ triweekly), resulting in complete remission after six cycles and lasting for more than 8 months; however, he developed interstitial pneumonia (Figure 1). Recently, features of immune infiltration were revealed for the first time, which contained pathological tissues from 31 cases, that PD-L1 expression of SHC was significantly higher than that of HCC, which also associated with poor prognosis in DFS $(\mathrm{HR}=5.036,95 \% \mathrm{CI}$ $1.382-18.354)$ and OS (HR=5.696, 95\% CI 1.473 22.081). ${ }^{47,50}$ It suggests that individualized analysis of immune check point and immunotherapy might be beneficial in PLSC patients, but adverse events still have to be carefully monitored.

There were some limitations in this study. (1) Fourteen cases of PLSC diagnosed using pathology were involved in this retrospective research. Selection bias could have existed due to only partial information derived from needle biopsies. (2) No immune-related examination had been performed on the 14 cases. Reasons speculated includes lack of relevant information and economic consideration. (3) The follow-ups were difficult because of the rapid progression and short survival periods in most cases, especially affecting the exact times of recurrences. (4) A total of 61 cases from ten studies were deleted in the analyses of necrosis proportions, satellite nodules, metastasis, and recurrences because of incomplete information reported in the original studies. This may have affected the accuracy of pathological features and clinical outcomes relevant to anti-tumor therapies.

\section{Conclusion}

PLSC is a rare, malignant, and aggressively invasive and metastatic tumor. PLSC is more prevalent in Asia. Misdiagnosis is possible due to nonspecific clinical findings. Without a standard treatment regimen, the prognosis is poor. Radical surgery is still recommended because it is the only treatment that can presently prolong survival times. Immunotherapy has achieved in several cases and should be considered.

\section{Data Sharing Statement}

Contact to the correspondent author Xu Li at jdyylx@jlu. edu.cn for data involved in the manuscript.

\section{Consent for Publication}

Written informed consents for publication were obtained from the patients or their lineal relations.

\section{Funding}

This work is supported by the grant from the National Natural Science Foundation of China (82071853).

\section{Disclosure}

The authors declare that there is no conflict of interest.

\section{References}

1. Bosman FT, Carneiro F, Hruban RH, et al. WHO Classification of Tumours of the Digestive System. 4th ed. Lyon: IARC press; 2010:196-261. 
2. Jernigan PL, Wima K, Hanseman DJ, et al. Natural history and treatment trends in hepatocellular carcinoma subtypes: insights from a national cancer registry. J Surg Oncol. 2015;112(8):872-876. doi: $10.1002 /$ jso. 24083

3. Albores-Saavedra J, Earl Hensonand D, Klimstra DS. Tumors of the gallbladder, extrahepatic bile ducts, and ampulla of vater. In: AFIP Atlas of Tumor Pathology. Series 3 Fascicle 8. American Registry of Pathology, 2000:130-133.

4. Li X, Li J, Liu K, Tan L, Liu Y. Sarcomatoid intrahepatic cholangiocarcinoma in a patient with poor prognosis: a case report and literature review. J Int Med Res. 2020;48(11):300060520969473. doi:10.1177/0300060520969473

5. Zhang N, Li Y, Zhao M, et al. Sarcomatous intrahepatic cholangiocarcinoma: case report and literature review. Medicine (Baltimore). 2018;97(39):e12549. doi:10.1097/MD.0000000000012549

6. Torbenson MS. Morphologic subtypes of hepatocellular carcinoma. Gastroenterol Clin North Am. 2017;46(2):365-391. doi:10.1016/j. gtc.2017.01.009

7. Kwon JH, Kang YN, Kang KJ. Carcinosarcoma of the liver: a case report. Korean $J$ Radiol. 2007;8(4):343-347. doi:10.3348/ kjr.2007.8.4.343

8. Okabayashi T, Shima Y, Iwata J, et al. Surgical outcomes for 131 cases of carcinosarcoma of the hepatobiliary tract. $J$ Gastroenterol. 2014;49(6):982-991. doi:10.1007/s00535-013-0882-2

9. Bin F, Chen Z, Liu P, Liu J, Mao Z. The clinicopathological and imaging characteristics of primary hepatic carcinosarcoma and a review of the literature. $J$ Hepatocell Carcinoma. 2020;7:169-180. doi:10.2147/JHC.S272768

10. Kim DK, Kim BR, Jeong JS, Baek YH. Analysis of intrahepatic sarcomatoid cholangiocarcinoma: experience from 11 cases within 17 years. World J Gastroenterol. 2019;25(5):608-621. doi:10.3748/ wjg.v25.i5.608

11. Mitra S, Gupta S, Dahiya D, Saikia UN, Rare A. Case of primary sarcomatous hepatocellular carcinoma without previous anticancer therapy. J Clin Exp Hepatol. 2017;7(4):378-384. doi:10.1016/j. jceh.2017.03.008

12. Yoshida N, Midorikawa Y, Kajiwara T, et al. Hepatocellular carcinoma with sarcomatoid change without anticancer therapies. Case Rep Gastroenterol. 2013;7(1):169-174. doi:10.1159/000350558

13. Lu J, Zhang J, Xiong XZ, et al. Primary hepatic sarcomatoid carcinoma: clinical features and prognosis of 28 resected cases. $J$ Cancer Res Clin Oncol. 2014;140(6):1027-1035. doi:10.1007/s00432-0141641-3

14. Chin S, Kim Z. Sarcomatoid combined hepatocellular-cholangiocarcinoma: a case report and review of literature. Int J Clin Exp Pathol. 2014;7(11):8290-8294.

15. Wang JP, Yao ZG, Sun YW, et al. Clinicopathological characteristics and surgical outcomes of sarcomatoid hepatocellular carcinoma. World J Gastroenterol. 2020;26(29):4327-4342. doi:10.3748/wjg. v26.i29.4327

16. Heimbach JK, Kulik LM, Finn RS, et al. AASLD guidelines for the treatment of hepatocellular carcinoma. Hepatology. 2018;67 (1):358-380. doi:10.1002/hep.29086

17. Boonsinsukh T, Viriyaroj V, Rookkachart T, Trongwongsa $T$. Intrahepatic sarcomatous cholangiocarcinoma: case report and review of the literature. Case Rep Surg. 2018;2018:3862575.

18. Srougi V, Gallucci FP, Mattedi RL, Srougi M. Carcinosarcoma of the bladder following local schistosomiasis infection. BMJ Case Rep. 2017;2017:bcr2016218642. doi:10.1136/bcr-2016-218642

19. Dahm P, Gschwend JE. Malignant non-urothelial neoplasms of the urinary bladder: a review. Eur Urol. 2003;44(6):672-681. doi:10.1016/S0302-2838(03)00416-0

20. Aparicio MA, Esteban C, Bengoechea O, Muñoz-Bellvís L. Primary carcinosarcoma of the liver: an unusual case with clearly separated epithelial and mesenchymal components. Rev Esp Enferm Dig. 2011;103(6):336-338. doi:10.4321/S1130-01082011000600014
21. Dongre A, Weinberg RA. New insights into the mechanisms of epithelial-mesenchymal transition and implications for cancer. Nat Rev Mol Cell Biol. 2019;20(2):69-84. doi:10.1038/s41580-018-0 080-4

22. Polyak K, Weinberg RA. Transitions between epithelial and mesenchymal states: acquisition of malignant and stem cell traits. Nat Rev Cancer. 2009;9(4):265-273. doi:10.1038/nrc2620

23. Wang QB, Cui BK, Weng JM, Wu QL, Qiu JL, Lin XJ. Clinicopathological characteristics and outcome of primary sarcomatoid carcinoma and carcinosarcoma of the liver. $J$ Gastrointest Surg. 2012;16(9):1715-1726. doi:10.1007/s11605-012-1946-y

24. Vajtai I, Vassella E, Hewer E, Kappeler A, Reinert MM. Sarcomatous evolution of oligodendroglioma ("oligosarcoma"): confirmatory report of an uncommon pattern of malignant progression in oligodendroglial tumors. Pathol Res Pract. 2012;208(12):750-755. doi:10.1016/j.prp.2012.09.009

25. De Sousa EMF, Vermeulen L, Fessler E, Medema JP. Cancer heterogeneity-a multifaceted view. EMBO Rep. 2013;14(8):686-695. doi:10.1038/embor.2013.92

26. Visvader JE, Lindeman GJ. Cancer stem cells: current status and evolving complexities. Cell Stem Cell. 2012;10(6):717-728. doi:10.1016/j.stem.2012.05.007

27. Fayyazi F, Nolte N, Oestmann O, Sattler S, Ramadori R, Radzun R. Carcinosarcoma of the liver. Histopathology. 1998;32(4):385-387. doi:10.1046/j.1365-2559.1998.0401j.X

28. Gu YJ, Zhu YY, Lu XY, Zhao Q, Cong WM. Hepatic carcinosarcoma: evidence of polyclonal origin based on microsatellite analysis. Pathol Res Pract. 2015;211(12):905-910. doi:10.1016/j. prp.2015.09.007

29. Matak A, Lahiri P, Ford E, et al. Stochastic phenotype switching leads to intratumor heterogeneity in human liver cancer. Hepatology. 2018;68(3):933-948. doi:10.1002/hep.29679

30. Wang T, Kong J, Yang X, Shen S, Zhang M, Wang W. Clinical features of sarcomatoid change in patients with intrahepatic cholangiocarcinoma and prognosis after surgical liver resection: a propensity score matching analysis. J Surg Oncol. 2020;121 (3):524-537. doi: 10.1002/jso.25815

31. Liu LP, Yu XL, Liang P, Dong BW. Characterization of primary hepatic carcinosarcoma by contrast-enhanced ultrasonography: a case report. World J Gastroenterol. 2014;20(6):1630-1634. doi:10.3748/wjg.v20.i6.1630

32. Pua U, Low SC, Tan YM, Lim KH. Combined hepatocellular and cholangiocarcinoma with sarcomatoid transformation: radiologic-pathologic correlation of a case. Hepatol Int. 2009;3 (4):587-592. doi:10.1007/s12072-009-9150-1

33. Shin HP, Jeon JW, Park JJ, et al. A case of leukemoid reaction in a patient with sarcomatous hepatocellular carcinoma. Korean J Hepatol. 2011;17(3):226-228. doi:10.3350/kjhep.2011.17.3.226

34. Hu B, Sang XT, Yang XB. Paraneoplastic leukemoid reaction in a patient with sarcomatoid hepatocellular carcinoma: a case report. World J Clin Cases. 2019;7(11):1330-1336. doi:10.12998/wjcc.v7. i11.1330

35. Bilgin M, Toprak H, Bilgin SS, Kondakci M, Balci C. CT and MRI findings of sarcomatoid cholangiocarcinoma. Cancer Imaging. 2012;12(3):447-451. doi:10.1102/1470-7330.2012.0036

36. Wang Y, Ming JL, Ren XY, et al. Sarcomatoid intrahepatic cholangiocarcinoma mimicking liver abscess: a case report. World J Clin Cases. 2020;8(1):208-216. doi:10.12998/wjcc.v8.11.208

37. Shi D, Ma L, Zhao D, et al. Imaging and clinical features of primary hepatic sarcomatous carcinoma. Cancer Imaging. 2018;18(1):36. doi:10.1186/s40644-018-0171-7

38. Seo N, Kim MJ, Rhee H. Hepatic sarcomatoid carcinoma: magnetic resonance imaging evaluation by using the liver imaging reporting and data system. Eur Radiol. 2019;29(7):3761-3771. doi:10.1007/ s00330-019-06052-8 
39. Yang Z, Lv K, Zhao Y, Pan M, Zhang C, Wei S. Sarcomatoid hepatocellular carcinoma mimicking hepatic abscess: a case report. Medicine (Baltimore). 2020;99(39):e22489. doi:10.1097/ MD.0000000000022489

40. Liu C, Wei S, Wu M, Kong W. Imaging features of ultrasound and contrast-enhanced ultrasound in primary hepatic carcinosarcoma: three cases report. Med Ultrason. 2019;21(4):487-490. doi:10.11152/mu-2002

41. Hwang S, Lee SG, Lee YJ, et al. Prognostic impact of sarcomatous change of hepatocellular carcinoma in patients undergoing liver resection and liver transplantation. J Gastrointest Surg. 2008;12 (4):718-724. doi:10.1007/s11605-007-0393-7

42. Jung GO, Park DE, Youn GJ. Huge subcapsular hematoma caused by intrahepatic sarcomatoid cholangiocarcinoma. Korean J Hepatobiliary Pancreat Surg. 2012;16(2):70-74. doi:10.14701/ kjhbps.2012.16.2.70

43. Yamamoto T, Kurashima Y, Ohata K, et al. Carcinosarcoma of the liver: report of a case. Surg Today. 2014;44(6):1161-1170. doi:10.1007/s00595-013-0612-7

44. Zhu SG, Li HB, Yuan ZN, et al. Achievement of complete response to nivolumab in a patient with advanced sarcomatoid hepatocellular carcinoma: a case report. World J Gastrointest Oncol. 2020;12 (10):1209-1215. doi:10.4251/wjgo.v12.i10.1209
45. Huang Z, Meng X, Liu Q. Simultaneous occurrence of sarcomatoid hepatocellular carcinoma and hepatocellular carcinoma. J Cancer Res Ther. 2015;11(3):665. doi:10.4103/0973-1482.140806

46. Yu Y, Zhong Y, Wang J, Wu D. Sarcomatoid hepatocellular carcinoma (SHC): a case report. World J Surg Oncol. 2017;15(1):219. doi:10.1186/s12957-017-1286-1

47. Luo C, Xin H, Yin D, et al. Characterization of immune infiltration in sarcomatoid hepatocellular carcinoma. Aging (Albany NY). 2021;13 (11):15126-15138. doi:10.18632/aging.203076

48. Xu J, Zhang Y, Jia R, et al. Anti-PD-1 antibody SHR-1210 combined with apatinib for advanced hepatocellular carcinoma, gastric, or esophagogastric junction cancer: an Open-Label, Dose Escalation and Expansion Study. Clin Cancer Res. 2019;25(2):515-523. doi:10.1158/1078-0432.CCR-18-2484

49. Zhao L, Yang Y, Gao Q. Efficacy and safety of nivolumab plus apatinib in advanced liver carcinosarcoma: a case report. Immunotherapy. 2019;11(8):651-656. doi:10.2217/imt-2018-0214

50. Morisue R, Kojima M, Suzuki T, et al. Sarcomatoid hepatocellular carcinoma is distinct from ordinary hepatocellular carcinoma: clinicopathologic, transcriptomic and immunologic analyses. Int J Cancer. 2021;149(3):546-560. doi:10.1002/ijc.33545

\section{Publish your work in this journal}

The Journal of Hepatocellular Carcinoma is an international, peerreviewed, open access journal that offers a platform for the dissemination and study of clinical, translational and basic research findings in this rapidly developing field. Development in areas including, but not limited to, epidemiology, vaccination, hepatitis therapy, pathology and molecular tumor classification and prognostication are all considered for publication. The manuscript management system is completely online and includes a very quick and fair peer-review system, which is all easy to use. Visit http://www.dovepress.com/ testimonials.php to read real quotes from published authors. 